\title{
ON THE STABILITY OF STATIONARY SOLUTIONS OF A LINEAR INTEGRO-DIFFERENTIAL EQUATION
}

\author{
A. YA. DOROGOVTSEV \\ Kiev Institute of Business and Technology \\ Blvd. T. Shevchenko, 4, 311 \\ 01033 Kiev-33 Ukraine \\ O. YU. TROFIMCHUK \\ Kiev University \\ Mechanics and Mathematics Department \\ Vladimirskay 64, 01033 Kiev-33 Ukraine
}

(Received October, 1999; Revised November, 2000)

In this paper the following two connected problems are discussed. The problem of the existence of a stationary solution for the abstract equation

$$
\epsilon x^{\prime \prime}(t)+x^{\prime}(t)=A x(t)+\int_{-\infty}^{t} E(t-s(x(s) d s+\xi(t), t \in \boldsymbol{R}
$$

containing a small parameter $\epsilon$ in Banach space $B$ is considered. Here $A \in$ $\mathcal{L}(B)$ is a fixed operator, $E \in C([0,+\infty), \mathcal{L}(B))$ and $\xi$ is a stationary process. The asymptotic expansion of the stationary solution for equation (1) in the series on degrees of $\epsilon$ is given.

We have proved also the existence of a stationary with respect to time solution of the boundary value problem in $B$ for a telegraph equation (6) containing the small parameter $\epsilon$. The asymptotic expansion of this solution is also obtained.

Key words: Stationary Solutions, Singular Perturbations, Telegraph Equation, Time-Stationary Solutions, Asymptotic Expansions.

AMS subject classifications: $34 \mathrm{G} 10,60 \mathrm{G} 20,60 \mathrm{H} 15,60 \mathrm{H} 99$.

\section{Introduction}

Let $(B\|\cdot\|)$ be a complex Banach space, $\overline{0}$ the zero element in $B$, and $\mathcal{L}(B)$ the Banach space of bounded linear operators on $B$ with the operator norm, denoted also by the symbol $\|\cdot\|$. For a $B$-valued function, continuity and differentiability refer to continuity and differentiability in the $B$-norm. For an $\mathcal{L}(B)$-valued function, continuity is the continuity in the operator norm. For operator $A$, the sets $\sigma(A)$ and $\rho(A)$ are its spectrum and resolvent set, respectively. 
In the following, we will consider random element son the same complete probability space $(\Omega, \Im, P)$. The uniqueness of a random process that satisfies an equation, is its uniqueness up to stochastic equivalence. We consider only $B$-valued random functions which are continuous with a probability of one. All equalities with random elements in this article are always equalities with a probability one. For a given equation, we consider only solutions which are measurable with respect to the right-hand side random process.

It is well known that the stationary solutions of difference and differential equations are steady with respect to various perturbations of the right-hand side and perturbation of coefficients. For example, see [5]. In the present work, it is shown that stability has a place with respect to perturbations such as degeneracy of the equation.

In the first part of this paper, we consider the following equation

$$
\epsilon x^{\prime \prime}(t)+x^{\prime}(t)=A x(t)+\int_{-\infty}^{t} E(t-s) x(s) d s+\xi(t), t \in \boldsymbol{R}
$$

containing a parameter $\epsilon$ in $B$. Here $A \in \mathcal{L}(B)$ is fixed operator, $\xi$ is a stationary process in $B$ and $E \in C([0,+\infty), \mathcal{L}(B))$ is a function satisfying the condition

$$
a:=\int_{0}^{+\infty}\|E(s)\| d s<+\infty
$$

We suppose that the following condition

$$
\sigma(A) \cap i \boldsymbol{R}=\emptyset
$$

holds. Under condition (2) the function

satisfies the inequality

$$
G(t):=\left\{\begin{array}{cc}
-e^{A t} P_{+}, & t<0 \\
e^{A t} P_{-}, & t>0
\end{array}\right.
$$

$$
b:=\int_{\boldsymbol{R}}\|G(s)\| d s<+\infty
$$

Here $P_{-}$and $P_{+}$are Riesz spectral projectors corresponding to the spectral sets $\sigma(A) \cap\{z \mid \operatorname{Re} z<0\}$ and $\sigma(A) \cap\{z \mid \operatorname{Re} z>0\}$, respectively.

Let $S$ be the class of all stationary $B$-valued processes $\{\xi(t): t \in \boldsymbol{R}\}$ which possess continuous derivatives of all orders on $\boldsymbol{R}$ with a probability one and such that, for some numbers $L=L_{\xi}>0, C=C_{\xi}>0, \delta>0$, the following inequalities

$$
\forall n \geq 0: \boldsymbol{E}\left\{\sup _{0 \leq s \leq \delta}\left\|\xi^{(n)}(s)\right\|\right\} \leq L C^{n}
$$

hold. The notations $\xi \in S(L, C, \delta)$ and $\xi \in S$ will be used. Then we have the following result. 
Theorem 1: Let $A \in \mathcal{L}(B)$ be an operator satisfying (2). Suppose that $\xi \in S$ and $a b<1$. Then there exists $\epsilon_{0}>0$ such that for every $\epsilon$ with $|\epsilon|<\epsilon_{0}$, the equation (1) has a stationary solution $x_{\epsilon} \in S$, which for every bounded subset $J$ of $\boldsymbol{R}$, satisfies

$$
\boldsymbol{E}\left(\sup _{s \in J}\left\|x_{\epsilon}(s)-y_{0}(s)\right\|\right) \rightarrow 0, \quad \epsilon \rightarrow 0,
$$

where $y_{0}$ is a unique stationary solution of the equation

$$
x^{\prime}(t)=A x(t)+\int_{-\infty}^{t} E(t-s) x(s) d s+\xi(t), t \in \boldsymbol{R} .
$$

The process $x_{\epsilon}$ is a unique solution of (1) in the class of all stationary connected processes in $S$.

This theorem is proved in Section 2. The method of proof uses a modification of the proof of Theorem 1 in [7] about the stability of stationary solutions for equation (1) with $E \equiv 0$.

Remark 1: The asymptotic expansion for a stationary solution of (1) is obtained.

Remark 2: The assumption (2) is equivalent to the existence of a unique stationary solution $\{x(t) \mid t \in R\}$ with $\boldsymbol{E}\|x(0)\|<+\infty$ of the equation

$$
x^{\prime}(t)=A x(t)+\xi(t), t \in \boldsymbol{R}
$$

for every stationary process $\{\xi(t) \mid t \in \boldsymbol{R}\}$ with $\boldsymbol{E}\|\xi(0)\|<+\infty$, see [3, pp. 201202].

Remark 3: The general approach to the analysis of the Cauchy problem for deterministic differential equations containing a small parameter leads to the appearance of boundary layer summands in the asymptotic expansion of solution [10]. These summands are absent in the asymptotic expansion of the stationary solution in the considered problem.

Remark 4: The problem of the existence of stationary solutions for difference and differential stochastic equations has been investigated by many authors. See, for example, monograph [1], surveys [2, 4] and article [6].

Corollary 1: Let $A \in \mathcal{L}(B)$ be an operator satisfying (1). Suppose that $\xi \in S$. Then there exists $\epsilon_{0}>0$ such that for every $\epsilon$ with $|\epsilon|<\epsilon_{0}$, the equation

$$
\epsilon x^{\prime \prime}(t)+x^{\prime}(t)=A x(t)+\xi(t), \quad t \in \boldsymbol{R}
$$

has a unique stationary solution $x_{\epsilon} \in S$, which, for every bounded subset $J$ of $\boldsymbol{R}$, satisfies

$$
\boldsymbol{E}\left(\sup _{s \in J}\left\|x_{\epsilon}(s)-x_{0}(s)\right\|\right) \rightarrow 0, \quad \epsilon \rightarrow 0
$$

where $x_{0}$ is a unique stationary solution of the equation

$$
x^{\prime}(t)=A x(t)+\xi(t), \quad t \in \boldsymbol{R} .
$$

The second part of this paper deals with the asymptotic expansion of the stationary with respect to time solution of a boundary value problem containing a small parameter. The following definition is necessary. 
Definition 1: A $B$-valued random function $u$ defined on $Q:=\boldsymbol{R} \times[0, \pi]$ is timestationary if

$$
\begin{gathered}
\forall t \in R \forall n \in N \forall\left\{\left(t_{1}, x_{1}\right), \ldots,\left(t_{n}, x_{n}\right)\right\} \subset Q \forall\left\{D_{1}, \ldots, D_{n}\right\} \subset \mathscr{B}(B): \\
P\left\{\bigcap_{k=1}^{n}\left\{\omega: u\left(\omega ; t_{k}+t, x_{k}\right) \in D_{k}\right\}\right\}=P\left\{\bigcap_{k=1}^{n}\left\{\omega: u\left(\omega ; t_{k}, x_{k}\right) \in D_{k}\right\}\right\}
\end{gathered}
$$

where $\mathfrak{B}(B)$ is the Borel $\sigma$-algebra of $B$.

Let

$$
C_{0}^{3}:=\left\{g:[0, \pi] \rightarrow C \mid g^{(k)}(0)=g^{(k)}(\pi)=0, k=0,1,2\right\} \cap C^{3}([0, \pi]) .
$$

Theorem 2: Let $A \in \mathcal{L}(B)$ be an operator satisfying the following condition

$$
\left\{k^{2}+i \alpha \mid k \in N, \alpha \in R\right\} \subset \rho(A) .
$$

Suppose that $g \in C_{0}^{3}$ and $\xi \in S$ with a number $\delta>0$ and $a b<1$. Then there exists $\epsilon_{0}>0$ such that for every $\epsilon$ with $|\epsilon|<\epsilon_{0}$, the boundary value problem

$$
\left\{\begin{array}{c}
\epsilon u_{t t}^{\prime \prime}(t, x ; \epsilon)+u_{t}^{\prime}(t, x ; \epsilon)-u_{x x}^{\prime \prime}(t, x ; \epsilon) \\
=A u(t, x ; \epsilon)+g(x) \xi(t), \quad t \in \boldsymbol{R}, x \in[0, \pi] \\
u(t, 0 ; \epsilon)=u(t, \pi ; \epsilon)=\overline{0}, t \in \boldsymbol{R}
\end{array}\right.
$$

has a unique time-stationary solution $u(\cdot, \cdot ; \epsilon)$ with

$$
\boldsymbol{E}\left(\sup _{0 \leq s \leq \delta, 0 \leq x \leq \pi}\|u(s, x ; \epsilon)\|\right)+\boldsymbol{E}\left(\sup _{0 \leq s \leq \delta, 0 \leq x \leq \pi}\left\|u_{t}^{\prime}(s, x ; \epsilon)\right\|\right)<+\infty
$$

which, for every $t \in \boldsymbol{R}$, satisfies

$$
\boldsymbol{E} \quad\left(\sup _{t \leq s \leq t+\delta, 0 \leq x \leq \pi}\|u(t, x ; \epsilon)-v(t, x)\|\right) \rightarrow 0, \quad \epsilon \rightarrow 0,
$$

where $v$ is the unique time-stationary solution of the following boundary value problem for a heat equation

with

$$
\left\{\begin{array}{c}
v_{t}^{\prime}(t, x)-v_{x x}^{\prime \prime}(t, x)=A v(t, x)+g(x) \xi(t), \quad t \in Q \\
v(t, 0)=v(t, \pi)=0, t \in \boldsymbol{R}
\end{array}\right.
$$

$$
\sup _{0 \leq x \leq \pi} \boldsymbol{E}\|v(0, x)\|<+\infty
$$

This theorem is proved in Section 3.

Remark 5: Condition (5) is a necessary and sufficient condition of the existence of a time-stationary solution for boundary value problem (7) [8].

Remark 6: Note that, if $\epsilon>0$, problem (6) is a boundary value problem for a hyperbolic equation and that, if $\epsilon=0$, we have a boundary value problem for a parabolic equation.

Remark 7: The study of the asymptotic behavior of a solution $u(\cdot, \cdot ; \epsilon)$ of the telegraph equation from (6) as $\epsilon \rightarrow 0+$ has also physical sense [9]. 


\section{Asymptotic Expansion of the Stationary Solution of Equation (1)}

In order to prove Theorem 1, a few lemmas will be needed.

Lemma 1: Let $A \in \mathcal{L}(B)$ be an operator satisfying (2). Suppose that $\xi \in S$. Then the equation

$$
x^{\prime}(t)=A x(t)+\xi(t), t \in \boldsymbol{R}
$$

has a unique stationary solution $x \in S$, which can be presented in the form

$$
x(t)=\int_{\boldsymbol{R}} G(t-s) \xi(s) d s=\int_{\boldsymbol{R}} G(s) \xi(t-s) d s, t \in \boldsymbol{R} .
$$

Proof: This is the corollary of Theorem 1 in [3, pp. 201-202].

Lemma 2: Let $A \in \mathcal{L}(B)$ be an operator satisfying (2). Suppose that $\xi \in S$. The following two statements are equivalent:

(i) A stationary process $x \in S$ is a unique stationary solution of the equation (3).

(ii) A stationary process $x \in S$ is a unique stationary solution of the equation

$$
x(t)=\int_{\boldsymbol{R}} G(t-s) \int_{-\infty}^{s} E(s-u) x(u) d u d s+\int_{\boldsymbol{R}} G(t-s) \xi(s) d s, t \in \boldsymbol{R} .
$$

Proof: The result is a consequence of Lemma 1 .

Lemma 3: Let $A \in \mathcal{L}(B)$ be an operator satisfying (2) and ab $<1$. Suppose that $\xi$ is a stationary process in $B$, which, for some $\delta>0$, satisfies

$$
\boldsymbol{E}\left(\sup _{0 \leq t \leq \delta}\|\xi(t)\|\right)<+\infty
$$

Then the equation (8) has a unique stationary solution $x$, which satisfies

$$
\boldsymbol{E}\left(\sup _{0 \leq t \leq \delta}\|x(t)\|\right)<+\infty
$$

Proof: Let $S_{0}$ be the class of all stationary connected $B$-valued processes $x$ which are stationary connected with $\xi$ and, for given $\delta>0$, satisfy (9). Let us introduce the operator

Then $T x \in S_{0}$ and

$$
(T x)(t):=\int_{\boldsymbol{R}} G(t-s) \int_{-\infty}^{s} E(s-u) x(u) d u d s+\int_{\boldsymbol{R}} G(t-s) \xi(s) d s, t \in \boldsymbol{R} .
$$

$$
\boldsymbol{E}\left(\sup _{0 \leq t \leq \delta}\|(T x)(t)-(T y)(t)\|\right) \leq a b \boldsymbol{E}\left(\sup _{0 \leq t \leq \delta}\|x(t)-y(t)\|\right)
$$

therefore $T$ is a continuous operator on $S_{0}$. Set

then $x_{0} \in S_{0}$ and

$$
x_{0}(t):=\int_{\boldsymbol{R}} G(t-s) \xi(s) d s, \quad t \in \boldsymbol{R}
$$




$$
\boldsymbol{E}\left(\sup _{0 \leq t \leq \delta}\left\|x_{0}(t)\right\|\right) \leq b \boldsymbol{E}\left(\sup _{0 \leq t \leq \delta}\|\xi(t)\|\right)
$$

Introduce the sequences of random processes

It is clear that

$$
x_{0}, x_{1}:=T x_{0}, x_{2}:=T x_{1}, \ldots, x_{n}:=T x_{n-1}, \ldots
$$

and for every $t \in \boldsymbol{R}$

$$
x_{n} \in S_{0}, n \in N ; x_{n+1}=T x_{n}, n \geq 0
$$

$$
\begin{aligned}
& \boldsymbol{E}\left\|x_{n+1}(t)-x_{n}(t)\right\| \leq \boldsymbol{E}\left(\sup _{t \leq s \leq t+\delta}\left\|x_{n+1}(s)-x_{n}(s)\right\|\right) \\
& \qquad \leq a(a b)^{n+1} \boldsymbol{E}\left(\sup _{0 \leq t \leq \delta}\|\xi(s)\|\right), n \geq 0 .
\end{aligned}
$$

Hence, the series

$$
x(t):=x_{0}(t)+\left[x_{1}(t)-x_{0}(t)\right]+\ldots+\left[x_{n}(t)-x_{n-1}(t)\right]+\ldots
$$

converges with a probability one for every $t \in \boldsymbol{R}$ and this convergence is uniform over the bounded subset of $\boldsymbol{R}$ with a probability one. By continuity of $T$ we have $x=T x$. The solution $x$ of $(8)$ is unique.

Lemma 4: Let $A \in \mathcal{L}(B)$ be an operator satisfying (2) and ab $<1$. Suppose that $\xi$ is a stationary process in $B$, which, for some $\delta>0$, satisfies

$$
\boldsymbol{E}\left(\sup _{0 \leq t \leq \delta}\|\xi(t)\|\right)<+\infty
$$

Then equation (3) has a unique stationary solution $x$, which satisfies (9).

Proof: The result is an immediate consequence of Lemma 2 and Lemma 3.

Set $c:=(1-a b)^{-1}$.

Lemma 5: Let $A \in \mathcal{L}(B)$ be an operator satisfying (2) and ab $<1$. Suppose that $\xi \in S(L, C, \delta)$. The equation (3) has a unique stationary solution $x \in S(b c L, C, \delta)$.

Proof: We return to the proof of Lemma 3 where the stationary solution $x$ for equation (3) was given. From the inclusion $\xi \in S(L, C, \delta)$ and representation

it follows that

$$
\begin{gathered}
x_{0}(t)=\int_{\boldsymbol{R}} G(s) \xi(t-s) d s, t \in \boldsymbol{R} \\
x_{0}^{(k)}(t):=\int_{\boldsymbol{R}} G(s) \xi^{(k)}(t-s) d s, \quad t \in \boldsymbol{R}
\end{gathered}
$$

for every $k \geq 0$ and $x_{0} \in S(b L, C, \delta)$. For the process $x_{1}-x_{0}$, we have

$$
x_{1}(t)-x_{0}(t)=\int_{\boldsymbol{R}} G(u) \int_{0}^{+\infty} E(v) x_{0}(t-u-v) d u d v, t \in \boldsymbol{R} .
$$

Hence, for every $k \geq 0$, we have

$$
x_{1}^{(k)}(t)-x_{0}^{(k)}(t)=\int_{\boldsymbol{R}} G(u) \int_{0}^{+\infty} E(v) x_{0}^{(k)}(t-u-v) d u d v, t \in \boldsymbol{R},
$$


and $\left(x_{1}-x_{0}\right) \in S\left(a b^{2} L, C, \delta\right)$. By induction, we find

$$
\left(x_{n}-x_{n-1}\right) \in S\left(b(a b)^{n} L, C, \delta\right), n \geq 1 .
$$

Therefore,

Lemma 5 is proved.

$$
x \in S(b c L, C, \delta)
$$

Proof of Theorem 1: Let $\xi \in S(L, C, \delta)$. We shall construct the asymptotic expansion for a solution of (1) in the following way. From Lemma 5, equation (3) has a unique stationary solution $y_{0} \in S(b c L, C, \delta)$. Note that $y_{0}^{\prime \prime} \in S\left(b c L C^{2}, C, \delta\right)$. Let $y_{1}$ be a unique stationary solution for equation

$$
y_{1}^{\prime}(t)=A y_{1}(t)+\int_{-\infty}^{t} E(t-s) y_{1}(s) d s-y_{0}^{\prime \prime}(t), t \in \boldsymbol{R}
$$

This solution exists from Lemma 5 and

$$
y_{1} \in S\left(b^{2} c^{2} L C^{2}, C, \delta\right)
$$

By analogy with $y_{1}$, let $y_{2}$ be a unique stationary solution for equation

$$
y_{2}^{\prime}(t)=A y_{2}(t)+\int_{-\infty}^{t} E(t-s) y_{2}(s) d s-y_{1}^{\prime \prime}(t), t \in \boldsymbol{R}
$$

For this solution, we have $y_{2} \in S\left(b^{3} c^{3} L C^{4}, C, \delta\right)$.

If the processes $y_{0}, y_{1}, \ldots, y_{n-1}$ for $n \geq 1$ are already constructed we will define process $y_{n}$ as a unique stationary solution of the equation

which satisfies

$$
y_{n}^{\prime}(t)=A y_{n}(t)+\int_{-\infty}^{t} E(t-s) y_{n}(s) d s-y_{n-1}^{\prime \prime}(t), \quad t \in \boldsymbol{R}
$$

$$
y_{n} \in S\left(b^{n+1} c^{n+1} L C^{2 n}, C, \delta\right) \text {. }
$$

It is clear that the processes $y_{n}, n \geq 0$ are stationary connected [3].

Set

$$
y_{\epsilon}(t):=\sum_{n=0}^{\infty} \epsilon^{n} y_{n}(t), t \in \boldsymbol{R} .
$$

Since

$$
\sum_{n=0}^{\infty}\left|\epsilon^{n}\right| \boldsymbol{E}\left(\sup _{t \leq s \leq t+\delta}\left\|y_{n}(s)\right\|\right) \leq \sum_{n=0}^{\infty}|\epsilon|^{n} \frac{b^{n+1} L C^{2 n}}{(1-a b)^{n+1}} \leq \frac{2 b L}{1-a b}
$$

for every $t \in \boldsymbol{R}$ and $|\epsilon| \leq \epsilon_{0}:=(1-a b) /\left(2 b C^{2}\right)$, the series for $y_{\epsilon}$ converges uniformly on bounded subsets of $\boldsymbol{R}$ with a probability one. This shows that $y_{\epsilon}$ is continuous on $\boldsymbol{R}$ with a probability one stationary process. 
By exactly the same arguments as those used above, we claim that the series for $y_{\epsilon}^{\prime}, y_{\epsilon}^{\prime \prime}$ are also absolutely and uniform convergent on bounded subsets of $\boldsymbol{R}$ with a probability one and we have

$$
\begin{gathered}
\epsilon y_{\epsilon}^{\prime \prime}(t)+y_{\epsilon}^{\prime}(t)=\sum_{n=0}^{\infty}\left(\epsilon^{n+1} y_{n}^{\prime \prime}(t)+\epsilon^{n} y_{n}^{\prime}(t)\right) \\
=\sum_{n=0}^{\infty}\left[\epsilon^{n+1}\left(A y_{n+1}(t)+\int_{-\infty}^{t} E(t-s) y_{n+1}(s) d s-y_{n+1}^{\prime}(t)\right)+\epsilon^{n} y_{n}^{\prime}(t)\right] \\
=\sum_{n=0}^{\infty} \epsilon^{n+1} A y_{n+1}+\sum_{n=0}^{\infty} \epsilon^{n+1} \int_{-\infty}^{t} E(t-s) y_{n+1} d s-\sum_{m=1}^{\infty} \epsilon^{m} y_{m}^{\prime}(t)+\sum_{n=0}^{\infty} \epsilon^{n} y_{n}^{\prime}(t) \\
=A\left(\sum_{m=1}^{\infty} \epsilon^{m} y_{m}(t)\right)+\int_{-\infty}^{t} E(t-s)\left(\sum_{m=1}^{\infty} \epsilon^{m} y_{m}(s)\right) d s+y_{0}(t) \\
=A y_{\epsilon}(t)+\int_{-\infty}^{t} E(t-s) y_{\epsilon}(s) d s-A y_{0}(t)-\int_{-\infty}^{t} E(t-s) y_{0}(s) d s+y_{0}^{\prime}(t) \\
=A y_{\epsilon}(t)+\int_{-\infty}^{t} E(t-s) y_{\epsilon}(s) d s+\xi(t), t \in R .
\end{gathered}
$$

Moreover, for every $t \in \boldsymbol{R}$, we have

$$
\boldsymbol{E}\left(\sup _{t \leq s \leq t+\delta}\left\|y_{\epsilon}(s)-y_{0}(s)\right\|\right) \leq \sum_{m=1}^{\infty}|\epsilon|^{m-1} \frac{b^{m+1} L C^{2 m}}{(1-a b)^{m+1}} \leq \frac{2 b^{2} L C^{2}}{(1-a b)^{2}} \epsilon
$$

if $|\epsilon| \leq \epsilon_{0}$.

To complete the proof of Theorem 1 we need show only the uniqueness. It is sufficient to prove the following fact. If $z$ is stationary connected with the process $x_{\epsilon}$ solution of (1), which satisfies

$$
\boldsymbol{E}\left(\sup _{0 \leq t \leq \delta}\|z(t)\|\right)<+\infty, \quad \boldsymbol{E} \quad\left(\sup _{0 \leq t \leq \delta}\left\|z^{\prime}(t)\right\|\right)<+\infty
$$

then $z=x_{\epsilon}$. We apply Lemma 4 in the following way. The difference $u$ : $=x_{\epsilon}-z$ is a stationary process which satisfies the equation

$$
\epsilon u^{\prime \prime}(t)+u^{\prime}(t)=A x(t)+\int_{-\infty}^{t} E(t-s) u(s) d s, \quad t \in \boldsymbol{R}
$$

and 


$$
\boldsymbol{E}\left(\sup _{0 \leq t \leq \delta}\|u(t)\|\right)<+\infty, \quad \boldsymbol{E}\left(\sup _{0 \leq t \leq \delta}\left\|u^{\prime}(t)\right\|\right)<+\infty
$$

Let us consider a Banach space $B^{2}$ of two vectors equipped with term-by-term linear operations and with the norm which is equal to the sum of the norms of the coordinates. Let

$$
\boldsymbol{u}(t):=\left(\begin{array}{c}
u^{\prime} \\
u
\end{array}\right), \mathbb{A}:=\left(\begin{array}{cc}
-\epsilon^{-1} & \epsilon^{-1} A \\
I & \Theta
\end{array}\right), \mathbb{E}:=\left(\begin{array}{cc}
\Theta & \epsilon^{-1} E \\
\Theta & \Theta
\end{array}\right)
$$

where $\Theta$ and $I$ are the zero operator and identity operator on $B$, respectively. Then the following equation in $B^{2}$

$$
\boldsymbol{u}^{\prime}(t)=A \boldsymbol{u}(t)+\int_{-\infty}^{t} \mathbb{E}(t-s) \boldsymbol{u} d s, \quad t \in \boldsymbol{R}
$$

is equivalent to the equation (11) in $B$. By direct computation we obtain that condition

$$
\sigma(A) \cap i \boldsymbol{R}=\emptyset
$$

is fulfilled if, for every $\alpha \in \boldsymbol{R}$, an operator $A-\left(i \alpha+\alpha^{2} \epsilon\right) I$ has a bounded inverse. For the justification of this assertion for all small $|\epsilon|$ it suffices to make use of condition (2) and the boundedness of operator $A$. Then, by Lemma 4, the equation (12) has a unique stationary solution and hence $\boldsymbol{u}(t)=\overline{0}, t \in \boldsymbol{R}$ with the probability of of one.

The proof is complete.

Remark 8: Let $B=\boldsymbol{R}$. It can be proven that the existence of expansion (10) for the solution of equation (4) leads to condition $\xi=C^{\infty}(\boldsymbol{R})$.

\section{Time-Stationary Solutions of the Boundary Value Problem for PDE Containing a Parameter}

Proof of Theorem 2: Let a process $\xi \in S(L, C, \delta)$ and a function $g \in C_{0}^{3}$ be given. Then, one can expand $g$ as

$$
g(x)=\sum_{k=1}^{\infty} g_{k} \sin k x, x \in[0, \pi] ; \quad\left\{g_{k}: k \geq 1\right\} \subset C,
$$

where the series on the right-hand since is uniformly convergent. Note that

$$
g_{k}=\frac{2}{k} \int_{0}^{\pi} g(x) \sin k x d x, k \geq 1
$$

Let $k \geq 1$ be fixed. From assumption (5) and Corollary 1, it follows that there is $\xi_{k}>0$ such that for every $\epsilon$ with $|\epsilon|<\epsilon_{k}$, the equation

$$
\epsilon v_{k}^{\prime \prime}(t ; \epsilon)+v_{k}^{\prime}(t ; \epsilon)+k^{2} v_{k}(t ; \epsilon)=A v_{k}(t ; \epsilon)+g_{k} \xi(t), t \in \boldsymbol{R}
$$


has a unique stationary solution $v_{k}(\cdot ; \epsilon)$ such that

$$
\boldsymbol{E}\left(\sup _{t \in J}\left\|v_{k}(t ; \epsilon)-v_{k}(t)\right\|\right) \rightarrow 0, \epsilon \rightarrow 0,
$$

where $v_{k}$ is a unique stationary solution of the equation

$$
v_{k}^{\prime}(t)+k^{2} v_{k}(t)=A v_{k}(t)+g_{k} \xi(t), t \in \boldsymbol{R}
$$

and $J$ is a bounded subset of $\boldsymbol{R}$. Moreover, for every $t \in \boldsymbol{R}$, we have

$$
\boldsymbol{E}\left(\sup _{t \leq s \leq t+\delta}\left\|v_{k}(s ; \epsilon)\right\|\right) \leq 2\left|g_{k}\right| L L_{1, k}
$$

and

$$
\boldsymbol{E}\left(\sup _{t \leq s \leq t+\delta}\left\|v_{k}(s ; \epsilon)-v_{k}(s)\right\|\right) \leq 2\left|g_{k}\right| L L_{1, k}^{2} C^{2}|\epsilon|
$$

if $|\epsilon| \leq \epsilon_{k}$, where

$$
L_{1, k}:=\int_{\boldsymbol{R}}\left\|G_{k}(s)\right\| d s<+\infty
$$

and $G_{k}$ is Green's function for operator $A-k^{2} I ; k \geq 1$. It follows from the properties of $G_{k}$ that

$$
L_{1, k} \leq \frac{\tilde{L}}{k^{2}-k_{0}^{2}}, \quad k>k_{0}
$$

where a number $\tilde{L}$ can be chosen to be independent of $k$.

Now we shall remark, that by virtue of boundedness of an operator $A$, the numbers $\epsilon_{k}, k \geq 1$ are identifiable and not depending on $k$. Really, let $k_{0}$ be the least natural number such that a spectrum of an operator $A-\left(\alpha^{2} \epsilon-k_{0}^{2}\right) I$ resides in the left half-plane. Then the spectrum of an operator $A-\left(\alpha^{2} \epsilon-k^{2}\right) I, k \geq k_{0}$ also resides in the left half-plane and it is possible to put $\epsilon_{0}:=\min \left\{\epsilon_{1}, \epsilon_{2}, \ldots, \epsilon_{k_{0}}\right\}>0$. Thus, for every $\epsilon,|\epsilon|<\epsilon_{0}$, all equations (13) have a unique stationary solution.

Let us consider the series

$$
u(t, x ; \epsilon):=\sum_{k=1}^{\infty} v_{k}(t ; \epsilon) \sin k x, \quad(t, x) \in Q
$$

for $|\epsilon| \leq \epsilon_{0}$. It follows from (14) and (16) that

$$
\sum_{k=1}^{\infty} \boldsymbol{E}\left(\sup _{t \leq s \leq t+\delta, 0 \leq x \leq \pi}\left\|v_{k}(t ; \epsilon) \sin k x\right\|\right) \leq \sum_{k=1}^{\infty} 2\left|g_{k}\right| L L_{1, k}<+\infty
$$

for every $t \in \boldsymbol{R}$ and $|\epsilon| \leq \epsilon_{0}$. This implies that the series (17) converges absolutely and uniformly on $[t, t+\delta] \times[0, \pi]$ with the probability one and the random function $u(\cdot, \cdot ; \epsilon)$ is a continuous, time-stationary with respect of time variable, random functions. In addition,

$$
\boldsymbol{E}\left(\sup _{0 \leq s \leq \delta, 0 \leq x \leq \pi}\|u(s, x ; \epsilon)\|\right)<+\infty .
$$


Using the above-mentioned reasoning, the following equalities are installed

$$
\begin{gathered}
u_{t}^{\prime}(t, x ; \epsilon):=\sum_{k=1}^{\infty} v_{k}^{\prime}(t, \epsilon) \sin k x, \\
u_{t t}^{\prime \prime}(t, x ; \epsilon):=\sum_{k=1}^{\infty} v_{k}^{\prime \prime}(t ; \epsilon) \sin k x \\
u_{x x}^{\prime \prime}(t, x ; \epsilon):=\sum_{k=1}^{\infty}\left(-k^{2}\right) v_{k}(t ; \epsilon) \sin k x
\end{gathered}
$$

for $(t, x) \in Q$ and uniform on $[t, t+\delta] \times[0, \pi]$ convergence with the probability one of an appropriate series for any $t \in R$ and $|\epsilon| \leq \epsilon_{0}$. We have also

$$
\boldsymbol{E}\left(\sup _{0 \leq s \leq \delta, 0 \leq x \leq \pi}\left\|u_{t}^{\prime}(s, x ; \epsilon)\right\|\right)<+\infty
$$

From (17), (18), and (13), it follows that

$$
\begin{gathered}
\epsilon u_{t t}^{\prime \prime}(t, x ; \epsilon)+u_{t}^{\prime}(t, x ; \epsilon)-u_{x x}^{\prime \prime}(t, x ; \epsilon) \\
=\sum_{k=1}^{\infty}\left(\epsilon v_{k}^{\prime \prime}(t ; \epsilon)+v_{k}^{\prime}(t ; \epsilon)+k^{2} v_{k}(t ; \epsilon)\right) \sin k x \\
=\sum_{k=1}^{\infty}\left(A v_{k}(t ; \epsilon)+g_{k} \xi(t)\right) \sin k x \\
=A u(t, x ; \epsilon)+g(x) \xi(t),(t, x) \in Q .
\end{gathered}
$$

Hence, the random function $u(\cdot, \cdot ; \epsilon)$ for $\epsilon$ with $|\epsilon|<\epsilon_{0}$ is a time-stationary solution of (6).

This solution is unique. To see this, we observe that for any $t \in R$, the elements $\left\{v_{k}(t ; \epsilon)\right\}$ are Fourier coefficients of $u(t, \cdot ; \epsilon) \in C^{2}([0, \pi], B)$ which determine $u(t, \cdot ; \epsilon)$ uniquely with the probability one. See, for example [3] for details. By Corollary 1 , the solutions of (13) are also determined uniquely with a probability one.

Similarly, by repeating the above arguments, we conclude that random function

$$
v(t, x):=\sum_{k=1}^{\infty} v_{k}(t) \sin k x, \quad(t, x) \in Q
$$

is a unique, stationary with respect to time variable, solution of (7) and

$$
\boldsymbol{E} \quad\left(\sup _{t \leq s \leq t+\delta, 0 \leq x \leq \pi}\|v(s, x ; \epsilon)\|\right)<+\infty
$$

for every $t \in \boldsymbol{R}$. Note that the random functions $u(\cdot, \cdot ; \epsilon),|\epsilon| \leq \epsilon_{0}$ and $v$ are timestationary connected.

Finally, let us consider the difference $u(\cdot, \cdot ; \epsilon)-v(\cdot, \cdot)$ for $|\epsilon|<\epsilon_{0}$. By Corollary 1 , the following inequalities

$$
\boldsymbol{E} \quad\left(\sup _{t \leq s \leq t+\delta, 0 \leq x \leq \pi}\|u(t, x ; \epsilon)=v(t, x)\|\right)
$$




$$
\leq \sum_{k=1}^{\infty} \boldsymbol{E}\left(\sup _{t \leq s \leq t+\delta}\left\|v(t ; \epsilon)-v_{k}(t)\right\|\right) \leq \sum_{k=1}^{\infty} 2 L\left|g_{k}\right| L_{1, k}^{2} C^{2}|\epsilon|
$$

hold.

Theorem 2 is proved.

\section{References}

[1] Arato, M., Linear Stochastic Systems with Constant Coefficients. A Statistical Approach, Springer-Verlag, Berlin-Heidelberg 1982.

[2] Bainov, D.D. and Kolmanovskii, V.B., Periodic solutions of stochastic functional equations, Math. J. Toyama Univ. 14:1 (1991), 1-39.

[3] Dorogovtsev, A. Ya., Periodic and Stationary Regimes of Infinite-Dimensional Deterministic and Stochastic Dynamically Systems, Vissha Shkola, Kiev 1992 (in Russian).

[4] Dorogovtsev, A. Ya., Periodic processes: a survey of results, Theory of Stoch. Proc. 2(18):3-4 (1996), 36-53.

[5] Dorogovtsev, A. Ya., Stability of stationary and periodic solution equations in Banach space, J. of Appl. Math and Stoch. Anal. 10:3 (1997), 249-255.

[6] Dorogovtsev, A. Ya., Periodic distribution solution for a telegraph equation, $J$. of Appl. Math and Stoch. Anal. 12:2 (1999), 121-131.

[7] Dorogovtsev, A. Ya., Stability of stationary solutions, Dokl. Acad. Nauk (Moscow) 369 (1999), 309-310.

[8] Dorogovtsev, A. Ya., Stationary solutions to boundary problem for the heat equations, Hiroshima Math. J. 30:2 (2000), 191-203.

[9] Tolubinskii, E.V., The Theory of Transposition Processes, Naukova Dumka, Kiev 1969 (in Russian).

[10] Vishik, M.I. and Lusternik, L.A., Regular degeneration and boundary layer for linear differential equations with a small parameter, Math. Surveys 12:5 (1957), 3-122 (in Russian). 


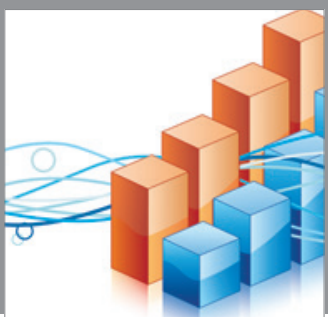

Advances in

Operations Research

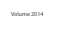

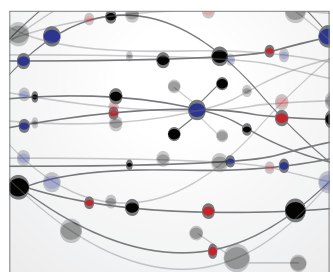

\section{The Scientific} World Journal
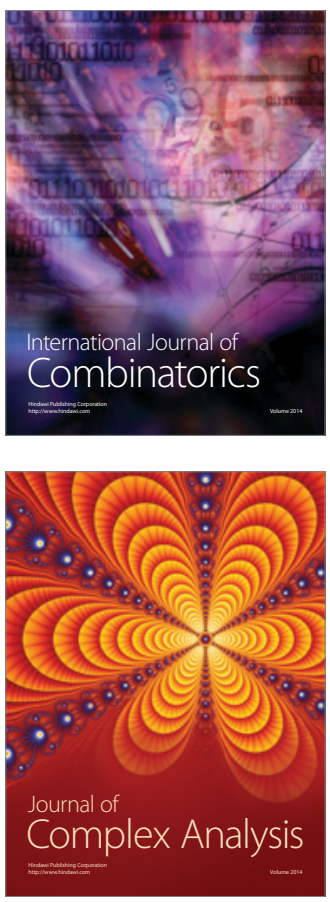

International Journal of

Mathematics and

Mathematical

Sciences
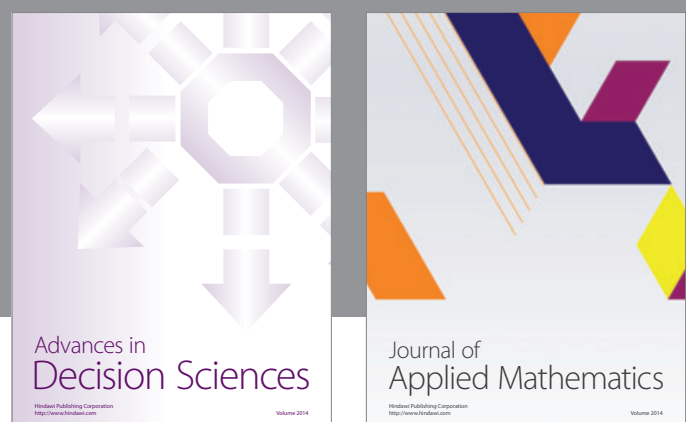

Journal of

Applied Mathematics
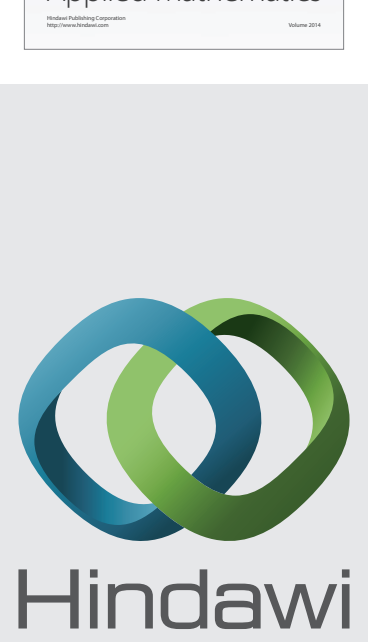

Submit your manuscripts at http://www.hindawi.com
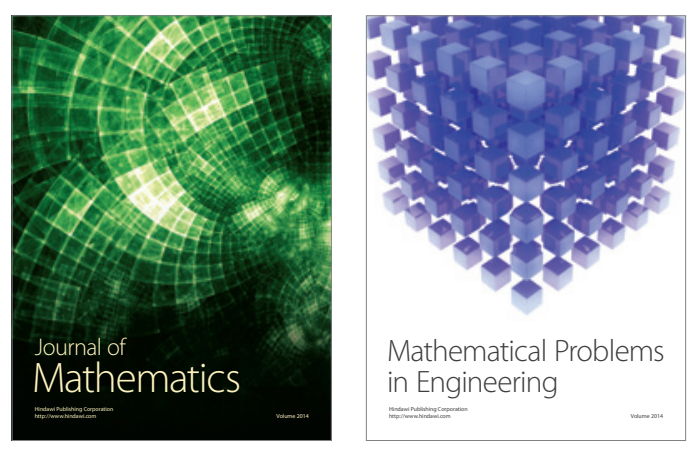

Mathematical Problems in Engineering
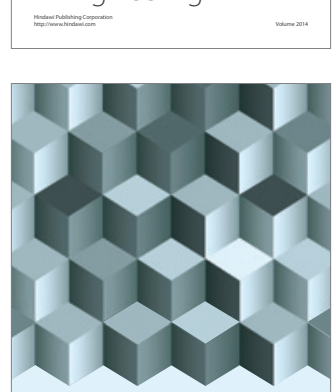

Journal of

Function Spaces
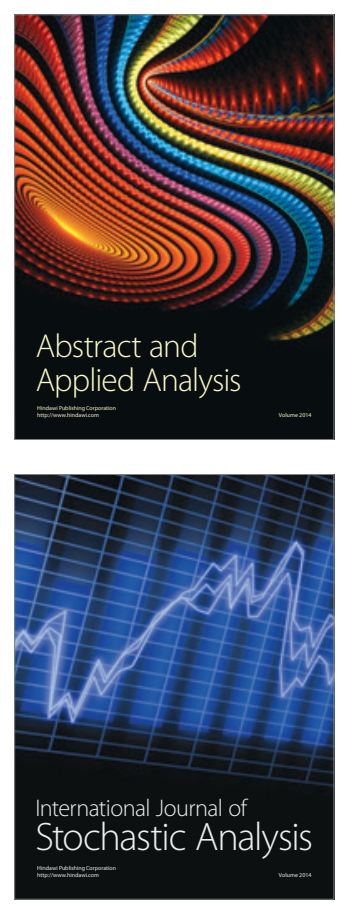

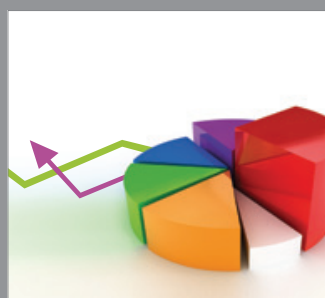

ournal of

Probability and Statistics

Promensencen
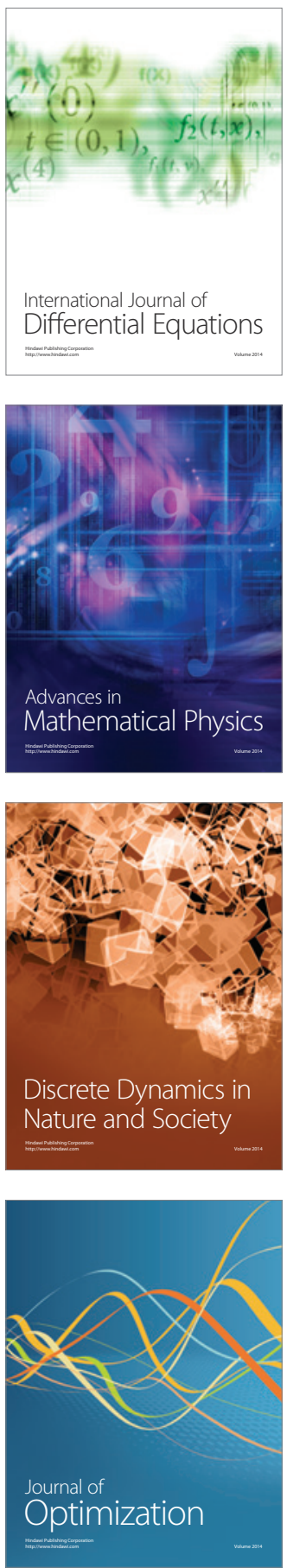